\title{
RELAXATION FUNCTION OF 3D XY MODEL $\dagger$
}

\author{
M.H. LEE $\ddagger$ and R. DEKEYSER \\ Instituut voor Theoretische Fysica, Katholieke Universiteit Leuven, B-3030 Heverlee, Belgium
}

\begin{abstract}
The Method of Mori has been extended in an effort to study the relaxation function. The difficulty posed by continued fractions has been in some special cases overcome to obtain exact solutions for the relaxation function. The physical significance of our solution is discussed with aid of the $X Y$ model.
\end{abstract}

Dynamical quantities of interacting manybody systems, such as scattering cross sections, conductivity, and others, can all be deduced from the relaxation function $\Xi(t)$, which describes how a given system relaxes in time $t$ after a perturbation has been in some suitable way removed at $t=0$. At present there is in spin dynamics, for example, considerable interest in calculating this quantity starting from model Hamiltonians. Of the various existing approaches, the one by Mori [1] developed from the ideas of Brownian motion appears to be based on the soundest ground. But there are in this approach certain inherent difficulties which have hitherto prevented it from being fully exploited. The approximations often employed to circumvent these difficulties are of questionable nature. Here we shall describe some of our preliminary advances made in developing the approach of Mori in connection with our study of the dynamics of the $X Y$ model.

Mori has shown that by writing

$\Xi(t)=\frac{1}{2 \pi \mathrm{i}} \int_{c} \mathrm{~d} z \mathrm{e}^{z t} \Xi(z)$,

$\Xi(z)$ is expressible as a continued fraction in terms of certain time independent correlation functions $\Delta_{n}$

$\Xi(z)=\frac{1}{z}+\frac{\Delta_{1}}{z}+\frac{\Delta_{2}}{z}+\frac{\Delta_{3}}{z}+\cdots$

These correlation functions are given by time derivatives as shown below: If $H$ is the Hamiltonian of the system and $c_{0}$ the order parameter of the system, e.g., $c_{0}(K)=\Sigma_{i} \mathrm{e}^{\mathrm{i} K \cdot R_{i}} S_{i}$, one can

† This research has been supported in part by grants from NATO (No. 1024) and Research Corporation.

$\ddagger$ Permanent address: Department of Physics, University of Georgia, Athens, Georgia 30602, USA. define the $n$th time derivative of $c_{0}$ as: $c_{n}=$ $\left[H, c_{n-1}\right]$ for $n \geqslant 1$. The correlation functions are then scalar products of $c_{n}$ 's $[1,2]$.

For nontrivial Hamiltonians, it is not a simple matter to obtain $c_{n}$ even for $n$ relatively small. Thus, one does not expect to have explicit forms of $\Delta_{n}$ except for possibly the first few of them. It is, nevertheless, evident that all correlation functions $\Delta_{n}$ exist as $n \rightarrow \infty$. This implies that the continued fraction (2) does not in general terminate, i.e., it is an infinite series; and one unavoidably encounters the mathematics of continued fractions. A standard procedure, first considered by Mori on physical grounds, is to replace $\Xi(z)$ by

$$
\Xi^{\prime}(z)=\frac{1}{z}+\frac{\Delta_{1}}{z}+\frac{\Delta_{2}}{z+z_{0}},
$$

where $z_{0}$ is a parameter. The above is a 3-pole approximation to a function with an infinite number of poles. Although this approximation has on occasion given some interesting results, it is all the same an unsound approximation as is discussed later. Here we show that the continued fraction can be made tractable without truncation if certain assumptions may be made on the behavior of $\Delta_{n}$ as $n \rightarrow \infty$. In some suitable limits these assumptions appear to be realizable in the $X Y$ model and we find some interesting physics concerning the excitations, dynamical modes, and others.

The continued fraction for $\Xi(z)$ may be expressed as [3]

$\Xi(z)=\lim _{n \rightarrow \infty} \operatorname{det} N_{n-1}(z) / \operatorname{det} D_{n}(z)$,

where $D_{n}(z)$ is an $n$-dimensional tridiagonal matrix whose elements $d_{i j}$ are: $d_{i i}=z$ and $d_{i j}=$ $-a_{j} \delta_{i j \pm 1}$, where $a_{n}=\mathrm{i} \Delta_{n}^{1 / 2}$; and $N_{n-1}(z)$ is the same matrix but without the first row and first 
column. With (1) we observe that $\Xi(t)$ may be determined if the zeros of $\operatorname{det} D_{n}(z)$ as $n \rightarrow \infty$ are first determined. We can rewrite $D_{n}(z)$ as (suppressing $n$ )

$D(z)=z I-\mathscr{D}$,

where $I$ is the unit matrix and $\mathscr{D}=-D(z=0)$. The requirement, det $D(z)=0$, implies that there exist a set of orthogonal eigenvectors $X_{r} r \rightarrow \infty$, satisfying the eigenvalue equations

$\mathscr{D} X_{r}=z_{r} X_{r}$

The eigenvectors represent the dynamical modes of the system. The zeros of det $D(z)$ are, thus, the eigenvalues of $\mathscr{D}$ and to obtain the eigenvalues $z_{r}$ we need to diagonalize the infinite dimensional matrix $\mathscr{D}$.

The infinite dimensional matrix $\mathscr{D}$ is diagonalizable under suitable conditions on $\Delta_{n}$ and, hence, $\Xi(t)$ may be in these cases explicitly determinable. Utilizing the properties of tridiagonal matrices we obtain the following 3term relation: For $n \geqslant 3$

$\operatorname{det} D_{n}=z \operatorname{det} D_{n-1}+\Delta_{n-1} \operatorname{det} D_{n-2}$.

The above is in the form of the general recurrence relation of orthogonal polynomials [4]. Thus, depending on $\Delta_{n}$, we can obtain the eigenvalues $z_{r}$ and the eigenvectors $X_{r}$ directly from orthogonal polynomials with the $n \rightarrow \infty$ limit appropriately taken afterwards.

In this way it is possible to determine $\Xi(t)$ for all $\left\{\Delta_{n}\right\}$ which belong to some set of orthogonal polynomials (e.g. Hermite, Gegenbauer). Now, the physical information is contained entirely in $\Delta_{n}$. Depending on these values of $\Delta_{n}$, we can obtain a whole new class of functions for $\Xi(t)$, whose time-dependent behavior can be different from the usually assumed exponential form.

We shall discuss here two simplest examples which are realizable in the $X Y$ model. (i) At high temperatures, if the coordination number of the system is large, we find [2] that $\Delta_{n}=\Delta$ for all $n$, where $\Delta$ is a constant. Then (7) becomes the recurrence relation for Tschebyshev polynomials of the second kind. Hence, the eigenvalues are the zeros of the polynomials, i.e. $z=$ $2 \mathrm{i} a \cos \theta$, where $0 \leqslant \theta \leqslant \pi$ and $a^{2}=\Delta$. The eigenvalues form a cut on the imaginary axis of the complex $-z$ plane from $z=+2 \mathrm{i} a$ to $z=-2 \mathrm{i} a$. The eigenvectors, which can be constructed from the Tschebyshev polynomials, are of plane wave form. The corresponding relaxation function obtained by integrating along the cut is

$\Xi(t)=2 J_{1}(2 a t) / 2 a t$,

where $J_{1}$ is the Bessel function of order 1 . The short and long time behaviors of $\Xi(t)$ are given by the asymptotic properties of the Bessel function:

$\Xi(t \rightarrow 0)=\cos a t$

and

$\Xi(t \rightarrow \infty)=\left(8 \pi a^{3} t^{3}\right)^{-1 / 2} \cos (2 a t-3 \pi / 4)$.

The short-time behavior agrees with a result earlier obtained via a mean field approximation valid for high frequencies [5]; and the long-time behavior is to our knowledge new. (ii) Also at high temperatures, if the system is one-dimensional, we find [2] that $\Delta_{1}=2 \Delta, \Delta_{n}=\Delta$ for $n \geqslant 2$. Then (7) becomes the recurrence relation for Tschebyshev polynomials of the first kind and the corresponding relaxation function is $\Xi(t)=$ $J_{0}(2 a t)$. The short and long time behaviors follow, again, from the asymptotic properties of the Bessel function. A fuller account of our result will appear in a future publication.

\section{References}

[1] H. Mori, Prog. Theor. Phys. (Kyoto) 33 (1965) 423, 34 (1965) 399.

[2] M.H. Lee, AIP Conf. Proc. 29 (1976) 472.

[3] M.H. Lee, Letts. Appl. Eng. Scis. 4 (1976) 63.

[4] G. Szegö, Orthogonal Polynomials (Am. Math. Soc., Providence, R.I., 1937) p. 42.

[5] M.H. Lee, Phys. Rev. B8 (1973) 3290. 